\title{
The spin-orbit alignment of visual binaries
}

\author{
A. B. Justesen and S. Albrecht \\ Stellar Astrophysics Centre, Department of Physics and Astronomy, Aarhus University, Ny Munkegade 120, 8000 Aarhus C, Denmark \\ e-mail: justesen@phys.au.dk
}

Received 10 August 2020 / Accepted 25 August 2020

\begin{abstract}
Context. The angle between the stellar spin-axis and the orbital plane of a stellar or planetary companion has important implications for the formation and evolution of such systems. A study by Hale (1994, AJ, 107, 306) found that binaries with separations $a \lesssim 30$ au are preferentially aligned while binaries on wider orbits are frequently misaligned.

Aims. We aim to test the robustness of the Hale study results by reanalysing the sample of visual binaries with measured rotation periods using independently derived stellar parameters and a Bayesian formalism.

Methods. Our analysis is based on a combination of data from the Hale study and newly obtained spectroscopic data from the Hertzsprung SONG telescope, combined with astrometric data from Gaia DR2 and the Washington Double Star Catalog. We combine measurements of stellar radii and rotation periods to obtain stellar rotational velocities $v$. Rotational velocities $v$ are combined with measurements of projected rotational velocities $v \sin i$ to derive posterior probability distributions of stellar inclination angles $i$. We determine line-of-sight projected spin-orbit angles by comparing stellar inclination angles with astrometric orbital inclination angles. Results. We find that the precision of the available data is insufficient to make inferences about the spin-orbit alignment of visual binaries. The data are equally compatible with alignment and misalignment at all orbital separations.

Conclusions. We conclude that the previously reported trend that binaries with separations $a \lesssim 30$ au are preferentially aligned is spurious. The spin-orbit alignment distribution of visual binaries is unconstrained. Based on simulated observations, we predict that it will be difficult to reach the sufficient precision in $v \sin i$, rotation periods, and orbital inclination required to make robust statistical inferences about the spin-orbit alignment of visual binaries.
\end{abstract}

Key words. binaries: visual - stars: solar-type - methods: data analysis - planets and satellites: dynamical evolution and stability protoplanetary disks

\section{Introduction}

The formation of binary stars is poorly understood. Disc fragmentation, turbulent fragmentation, and dynamical encounters may all play important roles in the formation of binary and higher-order systems (Bate 2019). If binary stars form preferentially via disc fragmentation, it is more likely that the stellar spins and protoplanetary discs of each star will be mutually aligned with each other and the binary orbit. On the other hand, if binary star formation occurs primarily through turbulent fragmentation or dynamical interactions, the stellar spin-axes in a binary system may be significantly misaligned with each other, the orbital plane, and the circumbinary disc (Bate et al. 2010; Bate 2018). An initially aligned protoplanetary disc may become misaligned with the stellar spin-axis due to secular interactions with an inclined binary companion (Batygin 2012). Hot Jupiters are often found in orbits misaligned with respect to the stellar spin (Winn et al. 2010; Albrecht et al. 2012). It is however unclear if these planets formed in misaligned protoplanetary discs or if misalignment occurred after planet formation due to high-eccentricity migration (see e.g. Dawson \& Johnson 2018). The spin-orbit alignment of binary stars is therefore important not only for constraining theories of star formation, but also for understanding the orbital evolution of planetary systems. If wide binary stars are found to be preferentially spin-orbit aligned, it is likely that protoplanetary discs are also well-aligned (Monin et al. 2007).
One of the most influential studies of spin-orbit alignment in binary stars was carried out by Hale (1994), who used measurements of rotation periods and projected rotational velocities, $v \sin i$, to constrain stellar inclination angles in visual binaries with known orbital inclinations. Hale found that binaries with semi-major axes smaller than 30-40 au are preferentially spin-orbit aligned while binaries in wider orbits are randomly aligned (see Fig. 1). Hale found that while double star systems are aligned below a separation of 30-40 au, this trend is not true for hierarchical multiple systems where spin-orbit misalignment is seen at all separations. Hale suggested that non-coplanarity may in fact decrease with separation in higher-order systems, the opposite trend seen for double star systems, although this trend is based on a small sample size.

Farbiash \& Steinitz (2004) studied the spin-spin alignment of a large sample of 1000 early-type binaries. They chose a sample of binaries (excluding triples or higher-order systems) in which they had measurements of $v \sin i$ of both components. They furthermore chose only main-sequence binaries of spectral type A9 or earlier. This was done to avoid slowly rotating stars or complications with loss of angular momentum during post-main-sequence evolution. They analysed the spin-spin alignment of the sample by comparing the $v \sin i$ of the two components in the binary with an artificially generated sample. They found that the stellar spin-axes of binary components are preferentially aligned at all orbital separations. The result of Farbiash \& Steinitz (2004) was challenged by Howe \& Clarke (2009), who analysed the same data and found that while the 


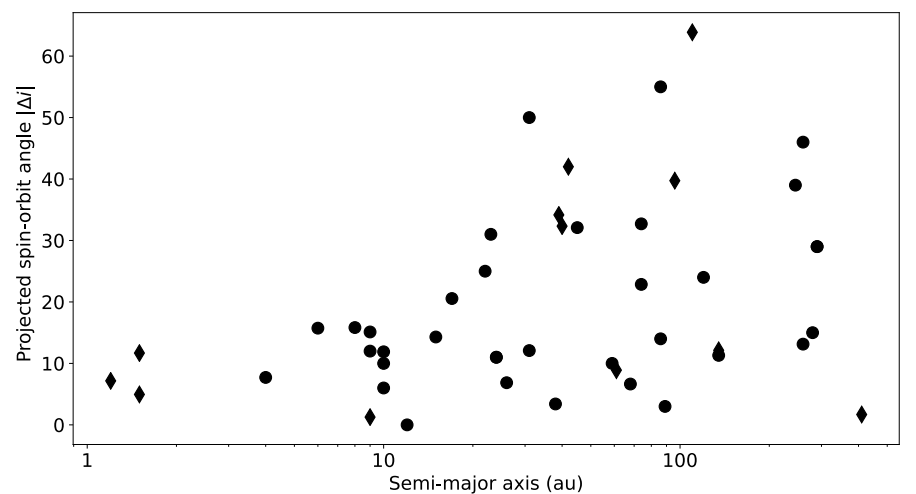

Fig. 1. Reprint of Fig. 2 from Hale (1994), although excluding three wide systems without orbital solutions. Systems plotted with circles have known rotation periods from time series or calibrated activity indices. Systems plotted with diamonds have rotational velocities estimated from an average $v \sin i$ based on spectral class or by assuming a Skumanich relation $(v \propto \sqrt{\tau})$ calibrated with the Hyades and using age estimates from Ca II fluxes or simply assuming an age of $3 \mathrm{Gyr}$. See Hale (1994) for details.

$v \sin i$ of components in close binaries with $a \lesssim 1$ au appear to be correlated (which they attribute to tidal synchronisation), the data are insufficient to constrain the alignment of binaries with separations wider than 1 au (i.e. the data are compatible with both aligned or misaligned configurations). Konopacky et al. (2012) studied a smaller sample of 11 VLM (Very Low Mass, spectral type M7 or later) binaries with $a \lesssim 10$ au. By analysing the $v \sin i$ of the individual components in the binaries, they find no evidence for preferential spin-spin alignment. They conclude that either the spin-axes of the components are misaligned or low-mass stars with similar spectral types rotate at significantly different speeds.

If a binary star is eclipsing, it is possible to constrain the projected obliquity by measuring the Rossiter-McLaughlin (RM) effect during the spectroscopic eclipse. The projected obliquity has been measured using the RM effect in just ten eclipsing binary systems ${ }^{1}$. Here we briefly summarise the results: Five binaries were analysed by Albrecht et al. (2007, 2009, 2011, 2013, 2014) as part of the BANANA (Binaries Are Not Always Neatly Aligned) Project. The BANANA Project found that the massive B-type binaries DI Herculis (Albrecht et al. 2009) and CV Velorum (Albrecht et al. 2013) have large projected obliquities of both components. The remaining binaries in the BANANA Project all have well-aligned configurations. Winn et al. (2011) measured the projected obliquity of the primary component in the planet-hosting eclipsing binary Kepler16 and found the system to be aligned, possibly due to tidal realignment. Finally, Sybilski et al. (2018) measured the projected obliquities of four eclipsing binaries and reported alignment for all systems except a marginal detection of a misaligned secondary component of AI Phe, although more data are needed to confirm that result. They note that tidal forces are predicted to have realigned all but one of their binaries. DI Herculis and CV

\footnotetext{
1 See Albrecht et al. (2011) for an overview of quantitative and qualitative RM analysis results up to 2011. The RM effect has additionally been measured for a few low-mass and unequal-mass binary systems as part of the EBLM project (Triaud et al. 2013; Gill et al. 2019; Hodžić et al. 2020), transiting brown dwarfs (Siverd et al. 2012) and hundreds of exoplanetary systems (see TEPCAT; Southworth 2011, www.astro.keele.ac.uk/jkt/tepcat/).
}

Velorum represent the only conclusive evidence for spin-orbit misalignment in close eclipsing binaries from the RM effect.

Zhou \& Huang (2013) analysed the photometric light curve of KOI-368, an eclipsing binary with an A-star primary and a M-dwarf companion. By analysing the asymmetric eclipse of the gravity-darkened host star, they find that the $P=110 \mathrm{~d}$ M-dwarf companion is in a significantly misaligned orbit.

There is evidence for both alignment and misalignment of protostellar and protoplanetary discs in binary systems. Jensen et al. (2004) measured the polarisation of near-infrared light in young binary systems and found evidence that suggested protoplanetary discs in binaries with separations 200-1000 au are typically aligned to within $20^{\circ}$, although the authors could not eliminate the possibility that their results are influenced by interstellar polarisation. More recently, it has become possible to directly image the discs of wide binaries. These observations reveal that disc-disc, disc-orbit and circumbinary disc misalignment are commonly found (Jensen \& Akeson 2014; Williams et al. 2014; Brinch et al. 2016; Aly et al. 2018; Kennedy et al. 2019; Mayama et al. 2020). Measurements of the orientation of outflow axes have similarly revealed that protostellar jets in young multiple protostar systems are commonly misaligned with respect to each other (Lee et al. 2016).

Tokovinin (2017) investigated the orbital alignment in hierarchical triple systems and found that the orbits of systems with outer separations less than $\sim 50$ au are preferentially aligned, while outer orbits with separations larger than 1000 au are misaligned with inner orbits. They note that the inner limit of $\sim 50$ au roughly matches the size of the circumstellar disc, suggesting that dissipative interactions with the disc have influenced the formation or evolution of close triples, acting to align these systems.

The results of Hale (1994) have been used to inform studies of binary formation and evolution. In particular, the preferential alignment of binaries with $a \lesssim 30$ au has been used as evidence that close binaries form via disc fragmentation while wider binaries form via turbulent core fragmentation (see e.g. reviews by Kratter 2011; Bate 2015). Recently, the Hale study has received considerable attention in the context of exoplanetary systems due to the possible role of inclined binary companions in the formation of misaligned hot Jupiters by disc tilting and high-eccentricity migration.

The conflicting results for the spin-orbit alignment of binaries, the importance of this result for binary and planetary formation, and the recent developments in the consistent application of the $v \sin i$ method motivated us to revisit the seminal Hale (1994) study. We therefore obtained high-resolution spectra of binary stars in the Hale study for a reanalysis of stellar parameters and projected rotational velocities. We reanalyse the Hale sample following a Bayesian approach and compute posterior probability distributions of stellar inclination angles. In Sect. 2 we present the Hale study and the sample of binary stars that we reanalyse in this work. In Sect. 3 we derive stellar parameters of the binary stars and compare our results to independent values. In Sect. 4 we present the projected spin-orbit alignment distribution of the binary sample based on our newly obtained data. In Sect. 5 we compare the observed spin-orbit distribution to simulated observations with various measurement uncertainties. We additionally discuss systematic errors and possible biases in the binary sample. Finally, we summarise our findings in Sect. 6.

\section{The visual binary sample}

The Hale (1994) study focuses on solar-type binary and multiple systems with primary spectral types F5V-K5V. The 
spectral range was chosen to ensure a sample of binaries for which the projected rotational velocities $v \sin i$ and the rotational modulation could be reliably measured. Binary stars with orbits of at least grade 3 ( 1 best -5 worst) in the Fourth Catalog of Orbits of Visual Binary Stars (VB83; Worley \& Heintz 1983) were chosen. Binaries in hierarchical multiple systems were also included. The study was restricted to nearby systems with accurate distances such that the true value of the semi-major axis could be obtained. The systems were further selected to encompass a wide range of orbital separations (1-10 $1000 \mathrm{au})$ and eccentricities. The Hale sample includes 86 stars in 73 stellar systems. 20 systems are triples or higher multiplicities.

In this work, we focus exclusively on binary star systems from the Hale sample (excluding stars in systems with higher multiplicities). We exclude higher-order systems due to the relatively small sample size. We further reject systems without orbital solutions (three systems) or known rotation periods (25 systems). Systems without known rotation periods are excluded due to concerns over the accuracy of rotation periods inferred from gyrochronology (see e.g. van Saders et al. 2016). This leaves us with a sample of 35 stars in 29 double star systems.

We briefly summarise the stellar and orbital parameters used by Hale. Hale compiled measurements of orbital elements, projected rotational velocities $v \sin i$, rotation periods $P_{\text {rot }}$ and stellar radii $R_{\star}$ from original work and various sources in the literature. Hale computed new orbits of six systems using measurements from literature combined with new astrometric observations. Hale combined literature values of $v \sin i$ with 32 new measurements. Stellar rotation periods were determined using one of three methods: rotational modulation of photometric light curves, time series analysis of spectroscopic Ca II fluxes, or calibration of chromospheric activity indices. The chromospheric flux ratio $R_{\mathrm{HK}}^{\prime}$ (defined as the ratio of chromospheric emission in the cores of the $\mathrm{Ca}$ II H and $\mathrm{K}$ lines to the total stellar bolometric emission) is correlated with the stellar rotation period (Noyes et al. 1984). A similar relation is seen for emission in $\mathrm{Mg}$ II lines. It is therefore possible to estimate stellar rotation periods from Ca II or Mg II fluxes by use of empirical activity-rotation relations. In the Hale binary sample, 17 stars have rotation periods calibrated from their Ca II flux ratios, 14 stars have rotation periods from time series and four stars have rotation periods calibrated from their Mg II flux ratio. Rotation periods and activity indices were compiled by Hale from the literature. Finally, stellar radii were obtained by Hale from calibrated colour-radius relations or from literature. Hale derived stellar inclination angles and corresponding uncertainties by error propagation of the relation

$i=\arcsin \left(\frac{v \sin i}{\left(2 \pi R_{\star}\right) / P_{\text {rot }}}\right)$.

Systems with values of $\sin i$ greater than unity had their inclination angles fixed to $90^{\circ}$. This is the case for 19 of the 35 stars in the Hale binary sample.

\section{Analysis}

We reexamine the Hale study by applying a more statistically robust Bayesian formalism as well as determining the accuracy of the stellar parameters used in Hale's study using highresolution spectroscopy. To establish the accuracy of the stellar parameters, we observed 25 stars in the Hale sample using the $1 \mathrm{~m}$ robotic Hertzsprung SONG Telescope (Andersen et al.
2014; Fredslund Andersen et al. 2019). We obtained spectra using the highest resolution configuration of $R=112000$ of the SONG telescope's echelle spectrograph (Grundahl et al. 2017). Here we describe the derivation of stellar parameters from the SONG spectra and compare our results with Hale and other independent estimates.

\subsection{Deriving stellar parameters}

We analyse SONG spectra with the open-source Python tool SpecMatch-emp (Yee et al. 2017). SpecMatch-emp derives stellar parameters of FGKM stars by comparison of the stellar spectrum with an empirical spectral library of well-characterised stars. SpecMatch-emp derives stellar parameters with a typical precision of $100 \mathrm{~K}$ in stellar effective temperatures, $15 \%$ in radii and 0.09 dex in metallicities. We analyse SONG spectra in the wavelength region 5000-5500 .

SpecMatch-emp does not derive calibrated projected rotational velocities $v \sin i$. To derive $v \sin i$, we compute synthetic spectra with effective temperatures, surface gravities and metallicities as determined by SpecMatch-emp. We synthesise spectra using the stellar synthesis framework iSpec (Blanco-Cuaresma et al. 2014) with the radiative transfer code SPECTRUM (Gray \& Corbally 1994) using ATLAS9 atmospheres (Kurucz 1979, 2005; Castelli \& Kurucz 2003), the VALD atomic line list (Kupka \& Dubernet 2011) and adopting Grevesse et al. (2007) solar abundances. We adopt the microturbulent velocity from the empirical relation implemented in iSpec. We do not apply instrumental, macroturbulent or rotational broadening at this stage, creating a sharp-lined template. Using our sharp-lined templates, we compute broadening functions (BFs) of each star (see Rucinski 1992, 1999, 2002). We fit the BF with a stellar line profile model including instrumental, macroturbulent and rotational broadening. We adopt Gaussian instrumental broadening corresponding to a resolution of $R=112000$ and macroturbulent broadening as determined from the empirical relation by Doyle et al. (2014). A few stars are slightly outside the 5200-6400 K temperature range of the Doyle et al. (2014) macroturbulence relation. In such cases we extrapolate the relation. We derive projected rotational velocities $v \sin i$ from best-fit line profiles. The formal uncertainty on $v \sin i$ as derived by fitting a line profile model to the BF is not an accurate estimate of the true uncertainty. The uncertainties are underestimated partly because the uncertainty in the other stellar parameters are neglected. Imperfect normalisation may also affect the BF and potentially the $v \sin i$. By computing the BF of various stars in the sample using different sharp-lined templates, we have found that our $v \sin i$ are generally precise to $1 \mathrm{~km} \mathrm{~s}^{-1}$ or better.

Although the systems in our sample are visual binaries, some systems have small sky-projected separations. We therefore visually inspected the BF of each spectrum to look for double peaks or asymmetries which would indicate that the spectrum is double-lined or blended. We found seven stars with spectra that indicated binarity. Since SpecMatch-emp is not designed to handle blended spectra, the stellar parameters derived from double-lined SONG spectra are not used in the analysis.

\subsection{Comparison of projected rotational velocities}

In Fig. 2 we plot a comparison between projected rotational velocities derived from SONG spectra, Hale and various literature values. We note that we have included the derived parameters from double-lined spectra in Figs. 2 and 3 only to 


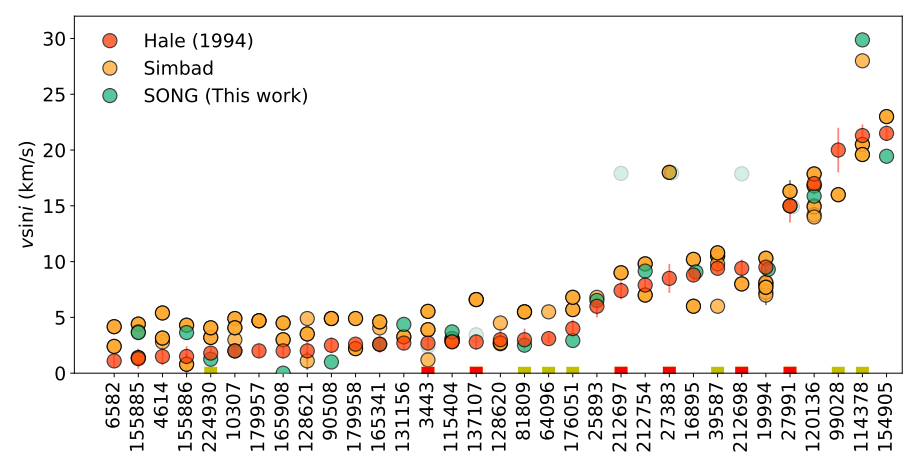

HD Catalogue Identifier

Fig. 2. Comparison of projected rotational velocities $v \sin i$ from Hale, Simbad (a collection of published values), and SONG spectra (this work). Yellow squares indicate stars with bright, nearby companions Red squares indicate stars identified in SONG spectra as double-lined. For visual clarity HD 113139 at $v \sin i=92 \mathrm{~km} \mathrm{~s}^{-1}$ is not shown HD 64096 does not have $v \sin i$ measurements from Simbad or SONG.

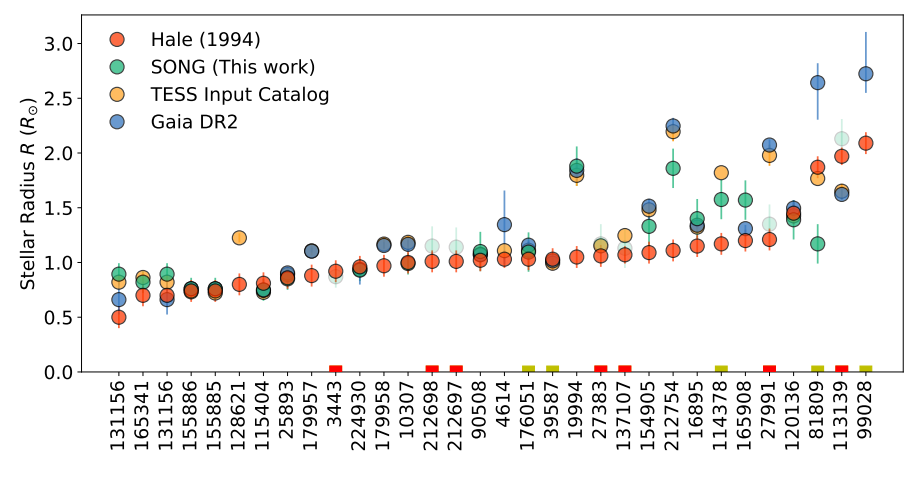

HD Catalogue Identifier

Fig. 3. Comparison of stellar radii from Hale, Gaia DR2, TESS Input Catalog, and SONG spectra (this work). Yellow squares indicate stars with bright, nearby companions. Red squares indicate stars identified in SONG spectra as double-lined. HD 6582, HD 64096 and HD 128620 do not have radius estimates from SONG, Gaia DR2 or TIC.

illustrate the effect of unmodelled binarity in the stellar spectra. The double-lined binaries HD 3443 and HD 137107 have clearly separated sets of spectral lines. For these stars we fitted both sets of lines in the BF and assumed that the deepest absorption lines belonged to the primary component. Since we study visual binaries, many stars will have nearby, bright companions that could affect the determination of stellar parameters. In Figs. 2 and 3, we therefore mark stars with companions with projected separations less than $3.5^{\prime \prime}$ and magnitude differences less than 3.25 ( $>5 \%$ flux difference) as listed in the Washington Double Star Catalog (WDS; Mason et al. 2019). We mark systems visually identified as double-lined with red squares and stars with close companions in the WDS with yellow squares. We find overall good agreement with projected rotational velocities of the 17 single-lined binaries observed with SONG and Hale with a median difference of $0.01 \pm 1.33 \mathrm{~km} \mathrm{~s}^{-1}$ (excluding the likely blended outlier HD 114378). It is clear that at $v \sin i<5 \mathrm{~km} \mathrm{~s}^{-1}$, it is difficult to obtain fractional uncertainties better than $\sim 20 \%$. At low rotational velocities, the rotational broadening, instrumental broadening, and macroturbulence all have similar magnitudes of a few kilometres a second. Literature values of $v \sin i$ are systematically larger than the $v \sin i$ derived in this work and Hale. This is likely due to neglect of macroturbulence in the literature values. The only significant disagreement between
Hale and SONG values is observed for HD 114378, likely due to blended lines in the SONG spectrum (as discussed in Sect. 3.5).

\subsection{Comparison of stellar radii}

In Fig. 3 we plot a comparison of stellar radii derived from SONG spectra, Hale, Gaia DR2 (Gaia Collaboration 2016, 2018), and the TESS Input Catalog (TIC, Stassun et al. 2019). We crossmatched the Hale sample with Gaia DR2 by querying the Gaia DR2 archive using HD Catalogue identifiers, which are resolved to coordinates and searched within a $3^{\prime \prime}$ radius. If multiple matches are found, we pick the brightest star within the search radius (since the stars in the Hale sample are all nearby, bright stars). We checked that we selected the correct matches for binaries with both components within the search radius. We found 24 matches with stellar radii in the Gaia DR2 archive. We similarly queried the TESS Input Catalog and found stellar radii for 26 stars in our sample. TESS and Gaia DR2 radii are not independent since TESS radii are derived using Gaia DR2 parallaxes with broadband photometry while Gaia DR2 radii are derived using the parallax and Gaia colours. We see good agreement for stars smaller than the Sun where SONG radii generally agree within $5 \%$ or better with Gaia DR2 and $10 \%$ or better with Hale. For a large fraction of stars larger than the Sun, there is significant disagreement between Hale radii and independent estimates with Hale radii being systematically smaller than other determinations. For stars larger than the Sun, SONG radii are generally accurate to within $\sim 15 \%$ of the Gaia DR2 radii, although some stars have larger disagreements. In Sect. 3.5, we discuss these stars in detail.

\subsection{Adopted parameters and uncertainties}

We adopt $v \sin i$ and stellar radii from single-lined SONG spectra where available, supplemented by $v \sin i$ from Hale (1994) and radii from Gaia DR2 or Hale (1994) for systems not observed by SONG or Gaia DR2. For stars with significant disagreement between Hale, SONG or Gaia DR2, we review the literature to determine which value to adopt, see Sect. 3.5. Based on the comparison between independently derived values, we adopt uncertainties of $1.3 \mathrm{~km} \mathrm{~s}^{-1}$ on $v \sin i, 5 \%$ fractional errors on stellar radii smaller than the Sun and $15 \%$ on radii larger than the Sun.

We adopt rotation periods from Hale (1994). Nearly all stars in the sample have rotation periods estimated from time series of $\mathrm{Ca}$ II fluxes or via activity-rotation relations using the Ca II activity index $R_{\mathrm{HK}}^{\prime}$. The Ca II fluxes used for these measurements were obtained at the Mount Wilson Observatory as part of the decades long survey started by Wilson $(1968,1978)$ and continued by Vaughan \& Preston (1980). Noyes et al. (1984) found that rotation periods estimated from $R_{\mathrm{HK}}^{\prime}$ are generally accurate to $15 \%$ or better. By analysing stars observed in multiple observing seasons, Donahue et al. (1996) found that rotation periods derived from time series of $\mathrm{Ca}$ II fluxes are generally accurate to $\sim 15 \%$. They attribute the scatter in rotation periods to the effect of surface differential rotation. We therefore adopt a fractional uncertainty of $15 \%$ on all rotation periods. The data from the Mount Wilson survey are the longest-running and most comprehensive set of consistent observations available for the stars in the sample. It is therefore difficult to find independent estimates of the rotation periods of the stars in our sample. Isaacson \& Fischer (2010) measured Ca II activity indices of 2630 stars at the Keck and Lick Observatories as part of the California Planet Search Program and estimated rotation periods using empirical activity-rotation relations. They observed six 
stars in our sample. We find a median difference of $25 \%$ between the rotation periods of Hale and Isaacson \& Fischer (2010), indicating that the uncertainty of rotation periods derived from $R_{\mathrm{HK}}^{\prime}$ could be larger than assumed, although this comparison is based on a very small sample size.

We use updated orbital inclinations from the Sixth Catalog of Orbits of Visual Binary Stars (Hartkopf et al. 2001, ORB6). We find generally excellent agreement between orbital inclinations in ORB6 and Hale (who used an earlier version of the same catalogue) with $\Delta i_{\text {orb }}=0.0_{-1.3}^{+0.97 \circ}$. Only five binaries differ by more than $5^{\circ}$ and no binary differ by more than $14^{\circ}$. We note that the good agreement between Hale and ORB6 is not necessarily indicative that the orbital inclination is well-determined. The median orbital period of the sample is $152 \mathrm{yr}$. For many binaries, only few measurements have been added to the ORB6 catalogue since 1994. Due to the long orbital periods, the solutions are therefore expected to be similar. The ORB6 catalogue does not provide robust uncertainties on orbital elements. Following Hale, we do not include the uncertainty of orbital inclinations in the analysis. The uncertainties on the projected spin-orbit angles therefore only reflect our knowledge of stellar inclination angles. We list adopted values, uncertainties, and references of all stars in the sample in Table 1.

\subsection{Notes on individual stars}

Here we discuss stars for which there is significant disagreement in the derived radii or projected rotational velocities from different sources.

$H D$ 19994. The Hale stellar radius at $R=1.05 \pm 0.1 R_{\odot}$ is significantly smaller than the radii of $R \sim 1.9 R_{\odot}$ predicted by Gaia DR2, TIC and this work. HD 19994 has been analysed interferometrically and found to have a radius of $R=1.898 \pm 0.070 R_{\odot}$ (van Belle \& von Braun 2009), in agreement with the analysis of the SONG spectrum. We adopt the interferometric radius.

$H D$ 81809. The spectroscopic SONG radius at $R=1.17 \pm$ $0.18 R_{\odot}$ is significantly smaller than other determinations at $R=$ $1.8-2.6 R_{\odot}$. HD 81809 has a close companion within $0.1^{\prime \prime}$ at a magnitude difference of $\Delta m=1.9$, potentially affecting values derived from both photometry and spectroscopy. The SONG spectrum does not appear double-lined from a visual inspection of the BF. The star is however listed as a spectroscopic binary on Simbad. Using resolved broadband photometry and the Gaia DR2 parallax, Egeland (2018) finds that HD 81809 is a sub-giant with a radius of $2.42 \pm 0.08 R_{\odot}$, consistent with the Gaia DR2 radius. We adopt the Gaia DR2 radius.

HD 212754. The Hale radius of $R=1.11 \pm 0.1 R_{\odot}$ is significantly smaller than other determinations of $1.9-2.2 R_{\odot}$. HD 212754 was recently discovered to be a single-lined spectroscopic binary with a period of 2.5 years (Griffin \& Suchkov 2003; Willmarth et al. 2016). Since the spectral lines of the companion are not visible it is unlikely to have affected the spectroscopic parameters. Based on the absolute visual magnitude, Willmarth et al. (2016) conclude that the star is evolved, in agreement with the spectroscopic SONG radius of $R=1.86 \pm$ $0.28 R_{\odot}$ which we adopt.

$H D 27991$. The radius listed in Gaia DR2 and TIC at $R \sim$ $2.1 R_{\odot}$ is significantly larger than the Hale and SONG radius of $R \sim 1.3 R_{\odot}$. This star has a companion at $0.1^{\prime \prime}$ with a magnitude difference of $\Delta m=0.74$. The BF of the SONG spectrum is visibly asymmetric, indicating contamination from the companion. We therefore cannot trust the spectroscopic radius.
Andrade (2019) analysed the binary system in a joint spectroscopic and astrometric analysis and derived a dynamical mass of $M=1.218 \pm 0.04 M_{\odot}$. They further find that the dynamical parallax is incompatible with the Gaia DR2 parallax, leading to incorrect radius estimates in Gaia DR2 and TIC. Unfortunately, the authors did not estimate the stellar radius. To determine the radius of HD 27991, we therefore derive stellar parameters from an isochrone analysis. HD 27991 is a member of the Hyades. We adopt an age of $625 \pm 50 \mathrm{Myr}$ and metallicity of $0.14 \pm 0.05$ dex (Perryman et al. 1998) and fit the dynamical mass, age and metallicity of HD 27991 to a grid of BaSTI isochrones (Hidalgo et al. 2018) using the Bayesian Stellar Algorithm BASTA (Silva Aguirre et al. 2015). We derive a stellar radius of $R=1.16 \pm 0.06 R_{\odot}$ in good agreement with the SONG and Hale radii. The SONG metallicity $[\mathrm{Fe} / \mathrm{H}]=0.13 \pm$ 0.09 and temperature $T_{\text {eff }}=6344 \pm 110 \mathrm{~K}$ are likewise in good agreement with the Hyades metallicity and isochrone-derived temperature of $6239 \pm 127 \mathrm{~K}$, indicating that the derived spectroscopic parameters are not significantly affected by the companion. We adopt the isochrone-derived radius.

$H D$ 114378. The Hale radius of $1.17 \pm 0.1 R_{\odot}$ is significantly smaller than the SONG and TIC radii of $R \sim 1.6-1.8 R_{\odot}$. The SONG $v \sin i$ of $30 \mathrm{~km} \mathrm{~s}^{-1}$ is significantly larger than the Hale value of $21 \mathrm{~km} \mathrm{~s}^{-1}$. HD 114378 has a companion at $\sim 0-0.5^{\prime \prime}$ (depending on the position in the 26 year orbit) with $\Delta m=0.68$. By close inspection, the BF of the SONG spectrum appear slightly asymmetric, indicating possible contamination from the companion. This slight asymmetry was missed in the initial visual inspection of the BF. Wright et al. (2011) derived a radius of $1.59 R_{\odot}$ by isochrone-fitting of broadband photometry but did not report the uncertainty. This radius is in agreement with the SONG radius of $1.58 \pm 0.18 R_{\odot}$. We therefore adopt the SONG radius and Hale $v \sin i$.

$H D$ 113139. The Hale radius of $\sim 2 R_{\odot}$ is larger than the Gaia DR2-based radius of $\sim 1.6 R_{\odot}$. HD 113139 has a companion at $0.9^{\prime \prime}$ with $\Delta m=2.86$. The BF of the SONG spectrum is clearly double-peaked. Kervella et al. (2019) derived a radius of $2.84 \pm 0.14 R_{\odot}$ using a brightness-colour relation. This radius is in significant disagreement with other determinations and is likely affected by contamination from the companion. The Hale radius is similarly derived from a colour-radius relation and may be contaminated. For this star we adopt the Gaia DR2 radius of $R=1.62 \pm 0.24 R_{\odot}$.

$H D$ 99028. The Hale radius of $2.1 \pm 0.1 R_{\odot}$ is significantly smaller than the Gaia DR2 radius of $2.72_{-0.17}^{+0.38} R_{\odot}$. Thévenin et al. (2006) measured an interferometric radius of $2.928 \pm 0.103 R_{\odot}$, consistent with the Gaia DR2 radius. We adopt the interferometric radius.

HD 155885 and HD 155886. HD 155885 and HD 155886 make up the bright binary $36 \mathrm{Oph}$. The two components are separated by $\sim 4^{\prime \prime}$ and are equally bright to a precision better than $1 \%$ $\left(V_{T}=5.12 \pm 0.01\right.$, Fabricius et al. 2002). The two components have equal radii, $v \sin i$ and rotation periods within the uncertainties of Hale and Gaia DR2, strongly indicating that the binary is equal-mass. We attempted to observed both components of 36 Oph with SONG. However, the similar brightness and small separation of the two components confused the automatic target acquisition of the robotic telescope. A visual inspection of the two SONG spectra reveal that they are identical at the level of the $\mathrm{S} / \mathrm{N}$ of the spectra. We therefore possibly observed the same target twice. It is however unclear if we observed HD 155885 or HD 155886. Using the SONG spectrum, we derive a radius 
Table 1. Adopted parameters of the binary sample.

\begin{tabular}{|c|c|c|c|c|c|c|c|c|c|c|c|}
\hline HD & $\begin{array}{l}v \sin i \\
\left(\mathrm{~km} \mathrm{~s}^{-1}\right)\end{array}$ & $\begin{array}{l}\sigma(v \sin i) \\
\left(\mathrm{km} \mathrm{s}^{-1}\right)\end{array}$ & $v \sin i$ Ref. & $\begin{array}{l}R_{\star} \\
\left(R_{\odot}\right)\end{array}$ & $\begin{array}{l}\sigma\left(R_{\star}\right) \\
\left(R_{\odot}\right)\end{array}$ & $R_{\star}$ Ref. & $\begin{array}{l}P_{\text {rot }} \\
\text { (d) }\end{array}$ & $\begin{array}{l}\sigma\left(P_{\text {rot }}\right) \\
(\mathrm{d})\end{array}$ & $P_{\text {rot }}$ Ref. & $\begin{array}{l}i_{\text {orb }} \\
\left({ }^{\circ}\right)\end{array}$ & $i_{\text {orb }}$ Ref. \\
\hline 3443 & 2.7 & 1.3 & 1 & 0.92 & 0.05 & 1 & 32.6 & 4.89 & 1 & 65.9 & 2 \\
\hline 4614 & 1.5 & 1.3 & 1 & 1.35 & 0.2 & 3 & 16.5 & 2.48 & 1 & 35.6 & 2 \\
\hline 6582 & 1.1 & 1.3 & 1 & 0.78 & 0.12 & 1 & 29.8 & 4.47 & 1 & 73.2 & 2 \\
\hline 10307 & 1.99 & 1.3 & 4 & 0.99 & 0.05 & 4 & 22.1 & 3.32 & 1 & 81.2 & 2 \\
\hline 16895 & 9.08 & 1.3 & 4 & 1.4 & 0.21 & 4 & 6.7 & 1.0 & 1 & 75.44 & 2 \\
\hline 19994 & 9.31 & 1.3 & 4 & 1.9 & 0.07 & 5 & 19.0 & 2.85 & 1 & 65.9 & 2 \\
\hline 25893 & 6.52 & 1.3 & 4 & 0.85 & 0.04 & 4 & 7.51 & 1.13 & 1 & 76.0 & 2 \\
\hline 27383 & 8.5 & 1.3 & 1 & 1.06 & 0.16 & 1 & 5.4 & 0.81 & 1 & 53.0 & 2 \\
\hline 27991 & 15.0 & 1.3 & 1 & 1.16 & 0.06 & 6 & 2.9 & 0.44 & 1 & 58.0 & 2 \\
\hline 39587 & 9.4 & 1.3 & 1 & 1.01 & 0.15 & 3 & 5.25 & 0.79 & 1 & 84.06 & 2 \\
\hline 64096 & 3.1 & 1.3 & 1 & 1.13 & 0.17 & 1 & 22.3 & 3.34 & 1 & 80.82 & 2 \\
\hline 81809 & 2.51 & 1.3 & 4 & 2.64 & 0.4 & 3 & 40.5 & 6.08 & 1 & 85.4 & 2 \\
\hline 90508 & 1.0 & 1.3 & 4 & 1.1 & 0.16 & 4 & 18.0 & 2.7 & 1 & 81.4 & 2 \\
\hline 99028 & 20.0 & 1.3 & 1 & 2.928 & 0.103 & 7 & 1.8 & 0.27 & 1 & 52.0 & 2 \\
\hline 113139 & 92.0 & 1.3 & 1 & 1.62 & 0.24 & 3 & 0.8 & 0.12 & 1 & 46.9 & 2 \\
\hline 114378 & 21.3 & 1.3 & 1 & 1.58 & 0.24 & 4 & 3.01 & 0.45 & 1 & 89.95 & 2 \\
\hline 115404 & 3.7 & 1.3 & 4 & 0.75 & 0.04 & 4 & 18.9 & 2.83 & 1 & 86.59 & 2 \\
\hline 120136 & 15.87 & 1.3 & 4 & 1.39 & 0.21 & 4 & 5.2 & 0.78 & 1 & 37.0 & 2 \\
\hline 128620 & 3.0 & 1.3 & 1 & 1.23 & 0.18 & 1 & 25.1 & 3.76 & 1 & 79.32 & 2 \\
\hline 128621 & 2.0 & 1.3 & 1 & 0.8 & 0.12 & 1 & 25.1 & 3.76 & 1 & 79.32 & 2 \\
\hline 131156 & 4.37 & 1.3 & 4 & 0.89 & 0.04 & 4 & 6.14 & 0.92 & 1 & 41.0 & 2 \\
\hline 137107 & 2.8 & 1.3 & 1 & 1.07 & 0.16 & 1 & 13.6 & 2.04 & 1 & 58.08 & 2 \\
\hline 154905 & 19.45 & 1.3 & 4 & 1.33 & 0.2 & 4 & 2.75 & 0.41 & 1 & 37.8 & 2 \\
\hline 155885 & 3.64 & 1.3 & 4 & 0.76 & 0.04 & 4 & 21.5 & 3.22 & 1 & 80.21 & 2 \\
\hline 165341 & 2.58 & 1.3 & 4 & 0.82 & 0.04 & 4 & 19.5 & 2.92 & 1 & 58.9 & 2 \\
\hline 165908 & 0.0 & 1.3 & 4 & 1.57 & 0.24 & 4 & 9.1 & 1.36 & 1 & 36.18 & 2 \\
\hline 176051 & 2.93 & 1.3 & 4 & 1.1 & 0.16 & 4 & 17.5 & 2.62 & 1 & 64.2 & 2 \\
\hline 179957 & 2.0 & 1.3 & 1 & 1.1 & 0.17 & 3 & 25.2 & 3.78 & 1 & 60.94 & 2 \\
\hline 179958 & 2.6 & 1.3 & 1 & 1.16 & 0.17 & 3 & 26.9 & 4.03 & 1 & 60.94 & 2 \\
\hline 212697 & 7.4 & 1.3 & 1 & 1.01 & 0.15 & 1 & 5.8 & 0.87 & 1 & 44.13 & 2 \\
\hline 212698 & 9.4 & 1.3 & 1 & 1.01 & 0.15 & 1 & 7.8 & 1.17 & 1 & 44.13 & 2 \\
\hline 212754 & 9.15 & 1.3 & 4 & 1.86 & 0.28 & 4 & 11.1 & 1.66 & 1 & 87.0 & 2 \\
\hline 224930 & 1.25 & 1.3 & 4 & 0.93 & 0.05 & 4 & 23.8 & 3.57 & 1 & 49.0 & 2 \\
\hline
\end{tabular}

References. (1) Hale (1994) and references therein; (2) ORB6 (Hartkopf et al. 2001); (3) Gaia DR2 (Gaia Collaboration 2016, 2018); (4) this work (spectroscopic); (5) van Belle \& von Braun (2009); (6) this work (isochrone); (7) Thévenin et al. (2006).

of $R=0.76 \pm 0.1 R_{\odot}$ and $v \sin =3.6 \pm 1 \mathrm{~km} \mathrm{~s}^{-1}$, in agreement with the stellar parameters of both HD 155885 and HD 155886 in Hale and Gaia DR2. Since the derived stellar parameters agree within the uncertainties in all cases, we simply adopt the SONG radius and $v \sin i$ as the stellar parameters of HD 155855 and reject HD 155886 from further study.

\section{Results}

Having obtained independent stellar parameters for the majority of the Hale binary sample and established their accuracy, we now estimate stellar inclination angles of stars in this sample. We compute stellar inclination angles using the Bayesian formalism of Masuda \& Winn (2020). We use normal likelihood functions of $v \sin i$ and construct likelihood functions of the rotational velocities $v$ from the normal distributions of stellar radii and rotation periods. We assume uniform priors in $\cos i$ and $v$. We compute posterior probability distributions of stellar inclination angles $p(\cos i \mid v, v \sin i)$ using Eq. (10) in Masuda \& Winn (2020). We compute projected spin-orbit angles as $\Delta i=i-i_{\text {orb }}$ with uncertainties derived from the $\cos i$ posterior probability distributions. We note that absolute value of $\Delta i$ is arbitrary in the sense that there is no difference between $\pm \Delta i$.

In Fig. 4 we plot the projected spin-orbit angle $\Delta i$ as a function of semi-major axis. It is immediately apparent that we do not reproduce a clear difference between binaries with separations smaller and larger than $\sim 30 \mathrm{au}$. We find that nearly all stars in the sample have poorly constrained stellar inclination angles, with a median size of the $68 \%$ confidence interval of $36^{\circ}$.

\section{Discussion}

The difference between the $\Delta i$ distribution derived in this work (Fig. 4) and Hale (Fig. 1) is mainly a result of the different radii used in the two works. We found that some stars in the Hale study have smaller radii than the ones derived from high-resolution spectroscopy or Gaia DR2 data. Another source of discrepancy comes from the derivation of stellar inclination angles. Hale derived stellar inclination angles by error propagation of Eq. (1), while we compute cos $i$ posteriors using Bayesian statistics. As shown by Masuda \& Winn (2020), simple error propagation (or simple Monte Carlo sampling) leads to incorrect $\cos i$ posteriors due to neglecting the correlation between 


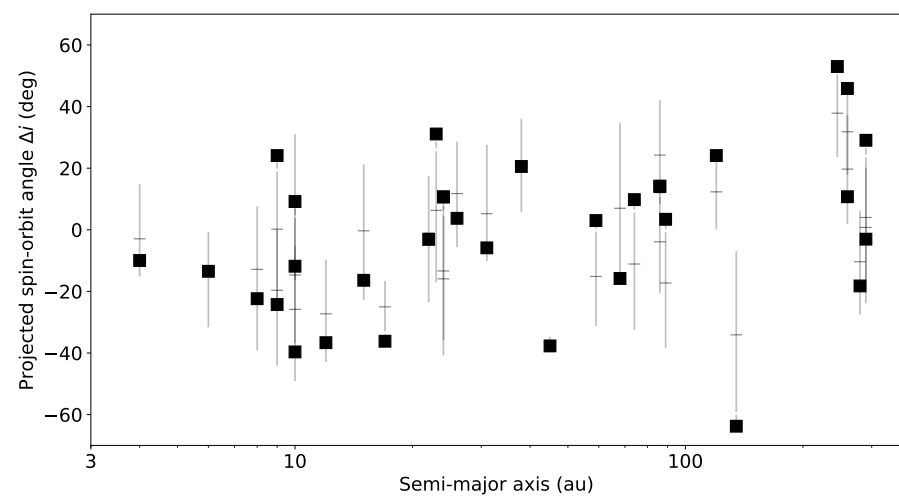

Fig. 4. Projected spin-orbit alignment of solar-type binaries. Full vertical lines indicate $68 \%$ confidence intervals. The median projected spin-orbit angle is marked with a short horizontal line. The most likely projected spin-orbit angle (using the mode of the posterior) is indicated with a black square. For systems in which $\Delta i_{\text {mode }}$ falls outside the $68 \%$ confidence interval (typically if $i_{\text {mode }}=90^{\circ}$ ), a dashed line indicates the extension of the confidence interval.

stellar rotational velocity $v$ and projected rotational velocity $v \sin i$. The exclusion of stars with rotation periods estimated from gyrochronology is unlikely to have affected the conclusions. Of the 25 stars without known rotation periods in the Hale study, nine stars are members of higher-order systems and two stars were excluded by Hale for being unreliable. Below $30 \mathrm{au}$, only four stars without rotation periods were excluded from this analysis compared to the Hale study. These stars were weighted lower in the Hale analysis to account for their increased uncertainty. As demonstrated in the following section, a large sample of well-characterised stars are needed to make robust inferences about the spin-orbit distribution.

\subsection{Simulated observations}

We compare the observed distribution of projected spin-orbit angles to the spin-orbit distribution of a randomly aligned population. This is done by generating samples of $\Delta i$ by drawing stellar and orbital inclination angles uniformly in $\cos i$. We compute the two-sided Kolmogorov-Smirnov (KS) statistic to test the hypothesis that the two distributions are drawn from the same underlying distribution ${ }^{2}$. We find a $p$-value of $p=0.4$, meaning that we cannot reject the null hypothesis with any significance.

We now test whether we could have detected if our binaries are all truly aligned. We simulate an aligned sample with characteristics similar to the Hale sample using the following procedure: We assume that the observed stellar radii $R_{\star}$, rotation periods $P_{\text {rot }}$, and orbital inclinations $i_{\text {orb }}$ as listed in Table 1 represent the true values of an aligned population of binaries. The $v \sin i$ of this aligned sample is computed as $v \sin i_{\text {orb }}=$ $\left(2 \pi R_{\star}\right) / P_{\text {rot }} \sin i_{\text {orb }}$. We then generate a set of observations by drawing values of $R_{\star}, P_{\text {rot }}$, and $v \sin i_{\text {orb }}$ using their estimated observational uncertainties as given in Table 1 (using $\sigma_{v \sin i}=$ $1.3 \mathrm{~km} \mathrm{~s}^{-1}$ for $v \sin i_{\text {orb }}$ ). Finally, using the artificially observed values (with the adopted observational uncertainties), we compute $\cos i$ posteriors and compute spin-orbit angles $\Delta i$ using the most likely stellar inclination angle $\Delta i=i_{\text {mode }}-i_{\text {orb }}$.

\footnotetext{
2 We note that the significance levels returned by the KS-test may not be reliable when comparing measurements with large uncertainties estimated from broad and asymmetric posteriors. We therefore caution against interpreting the $p$-values as precise estimators of significance.
}

We simulate 100 well-aligned samples and compare each sample with a randomly aligned sample using the KS-test. We find that our artificially observed aligned samples are statistically indistinguishable to randomly aligned samples with a mean KS-statistic of $p=0.3$. We therefore conclude that our data set is insufficient to make any conclusions about the spin-orbit alignment of binaries: Our data are compatible both with completely random alignment and with perfect alignment. With the adopted uncertainties of our $v \sin i$, radii and rotation periods, it is not possible to obtain stellar inclination angles of the required precision.

Having found that the current sample cannot be used to learn about spin-orbit alignment in binary stars, what kind of sample is required? As a first step we now investigate the needed precision to distinguish between a well-aligned and randomly aligned population. Even in a completely randomly aligned sample, the projected spin-orbit angle $|\Delta i|$ is less than $20^{\circ}$ for half of all systems and less than $60^{\circ}$ for $95 \%$ of systems. This is due to the fact that inclination angles are uniformly distributed in $\cos i$, not $i$. For stars with measurement uncertainties of $\sim 10 \%$ on $v$ and $v \sin i$, the flat $\cos i$ posterior results in large uncertainties on stellar inclination angles, making it difficult to distinguish between preferentially aligned and misaligned populations without relatively large samples of precisely characterised systems.

We simulated four binary samples, which we show in Fig. 5. All simulated populations of binaries are preferentially aligned at small separations and randomly aligned at larger separations. We model the projected spin-orbit alignment distribution of binaries with separations less than 40 au as a normal distribution with a standard deviation of $10^{\circ 3}$. We simulate 150 binaries loguniformly distributed over separations of $0.5-1000 \mathrm{au}$. Orbital inclinations are assumed to be uniformly distributed in $\cos i$. We do not include measurement uncertainty in orbital inclinations. We assign rotational velocities $v$ of binaries in our sample uniformly in the range $0-15 \mathrm{~km} \mathrm{~s}^{-1}$ or $15-50 \mathrm{~km} \mathrm{~s}^{-1}$, roughly approximating a solar-like and a F-type sample. We adopt normal likelihood functions with fractional uncertainties of either $15 \%$ and $5 \%$ on rotational velocities and absolute uncertainties of $2 \mathrm{~km} \mathrm{~s}^{-1}$ or $1 \mathrm{~km} \mathrm{~s}^{-1}$ on $v \sin i$. The upper two panels of Fig. 5 show the simulated solar-like sample. With uncertainties of $\sigma(v)=15 \%$ and $\sigma(v \sin i)=2 \mathrm{~km} \mathrm{~s}^{-1}$, we do not see any difference between close and wide binaries. Assuming optimistic uncertainties of $5 \%$ on rotational velocities and $1 \mathrm{~km} \mathrm{~s}^{-1}$ on $v \sin i$, the projected spin-orbit distribution shows a hint of increased scatter at large separations (although simple KS- or Anderson-Darling tests do not establish statistically significant differences between the two populations). Increasing the rotational velocities of the sample (lower two panels of Fig. 5) decreases the fractional uncertainty on $v \sin i$, thereby increasing the precision of the stellar inclination angles. With realistic uncertainties, the spin-orbit distribution similarly shows a hint of increased scatter at large separations, although with low significance. The final simulation (lower right panel) shows a fast rotating sample with optimistic uncertainties. In this case, the difference between close and wide binaries is finally clearly visible.

\footnotetext{
3 We note that the choice of a normal distribution is somewhat arbitrary. Fabrycky \& Winn (2009) describes the spin-orbit distribution as a Fisher distribution. However, the exact choice of the distribution is not important here.
} 

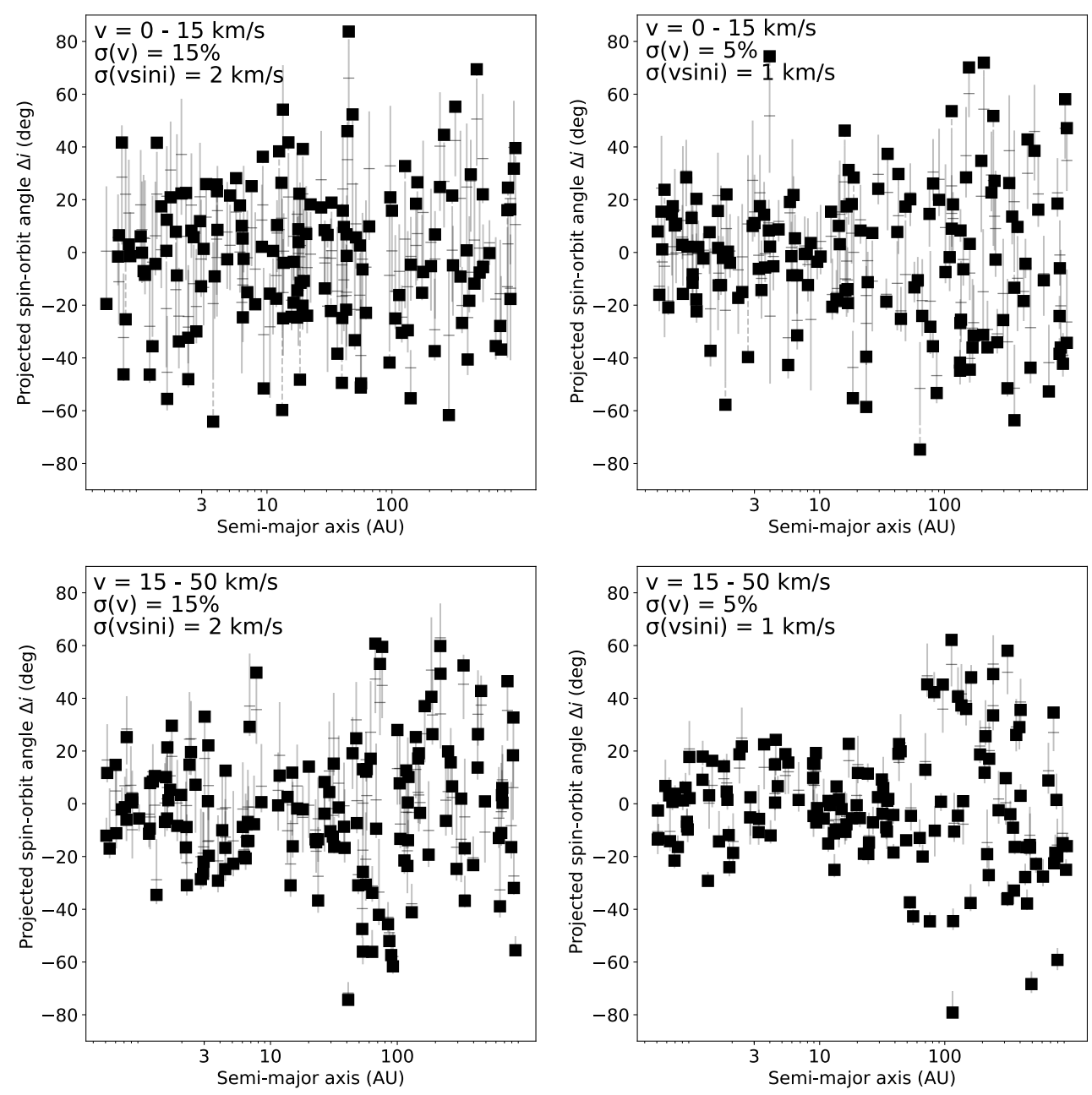

Fig. 5. Projected spin-orbit distributions of simulated binary samples. Rotational velocities of binaries in the sample are drawn from a uniform distribution with ranges indicate in each panel. We describe $v$ and $v \sin i$ as normal distributions with measurement uncertainties indicated in each panel.

\subsection{Systematic uncertainties and biases}

The derivation of the projected spin-orbit angle depends on precise and accurate measurements of the orbital inclination, stellar radius, stellar rotation period and projected rotational velocity. Each of these measurements may introduce difficult-to-quantify systematic uncertainties. The uncertainty on orbital inclination is neglected in this work. For wide binaries with sparsely sampled orbits, the uncertainty on the orbital inclination may be large.

Stellar rotation periods derived from chromospheric activity indices may be affected by a number of systematic uncertainties. Seasonal changes in the chromospheric activity of the star will affect the measured activity index. It is therefore important to use mean activity indices derived from measurements covering a large timeline. Other systematic error sources include large scatter in the empirical activity-rotation relation, stellar parameters outside calibrated range, differently calibrated activity indices, and stellar rotation affected by for example tidal spin-up or spindown due to close companions. For rotation periods determined from time series analysis, there is a risk of identifying harmonics or aliases of the true rotational signal as the rotation period. For photometric rotation periods, non-eclipsing close binaries with ellipsoidal variations may be mistaken for rotational modulation.
For hotter stars, there is a risk of confusing stellar rotation with stellar pulsations.

Projected rotational velocities depend on empirically calibrated macroturbulence. If the macroturbulence is incorrectly or inconsistently calibrated, $v \sin i$ values will be systematically under- or overestimated, at least for $v \sin i$ below a few $\mathrm{km} \mathrm{s}^{-1}$. Differential rotation has been neglected here but may affect the determination of both $v \sin i$ and $P_{\text {rot }}$ to a small amount.

The derived stellar radius may be significantly off if the evolutionary state of the star is wrongly classified. The derivation of precise radii of binary components with small sky-projected separations is complicated by blended photometry, potentially incorrect parallaxes and blended absorption lines.

In addition to systematic uncertainties, the binary sample will be biased due to various selection criteria and observational constraints. Some systems may have undetected companions, either planetary or stellar. Such companions could have complicated the evolution of the system, potentially obscuring trends in the binary spin-orbit distribution. By comparing stellar and orbital inclination angles, it is only possible to constrain the projected alignment along the line of sight. Since the sky-projected alignment of the stellar spin is unconstrained, it is possible that some systems with small projected spin-orbit angles are mis- 
aligned in 3D space. It is similarly not possible to distinguish between prograde and retrograde orbits.

A major difficulty is obtaining precise rotation periods for a range of spectral classes. Activity-rotation relations are only calibrated for solar-type stars. The correlation between rotation and chromospheric activity breaks down for stars hotter than $\sim 6200 \mathrm{~K}$ which have low chromospheric activity and shallow (or no) convective envelopes (Mamajek \& Hillenbrand 2008). It is similarly more difficult to obtain photometric rotation periods of hot stars. Stars with shallow convective envelopes or radiative envelopes do not produce star spots similar to cooler stars with convective envelopes. It is however possible to measure rotation periods from other phenomena such as chemical spots (photometric or spectroscopic) or magnetic field inhomogeneities (spectroscopic or polarimetric), although these methods are not widely applicable. Solar-like stars with rotation periods determined from rotational flux modulation are biased towards inclinations near $90^{\circ}$ due to the inclination-dependent amplitude of the star spot signal. This bias does not apply to rapidly rotating and magnetically active stars which often have polar star spots (Schuessler \& Solanki 1992).

\section{Conclusions}

We reanalysed a subset of the Hale sample using revised stellar and orbital parameters, partly based on new observations. We did not find evidence for the often-quoted statement that binary stars with separations less than 30 au are preferentially aligned or that binaries in wider orbits are preferentially misaligned. The trend observed in Hale (1994) is likely a result of underestimating the radii of some stars in the sample. Using a modern Bayesian formalism, we found that poorly constrained stellar inclination angles make inferences about the spin-orbit alignment of the current sample impossible (i.e. the data are compatible with both alignment and random orientation).

To date, only sparse observational data exist to constrain the spin-orbit alignment of binaries. Observations of a handful of protoplanetary discs in young binaries indicate that disc-disc misalignment may be common (Jensen \& Akeson 2014; Brinch et al. 2016). However, from measurements of polarised light there is similarly evidence for disc-disc alignment (Jensen et al. 2004). We do not know how often tidal forces realign such protoplanetary discs or if the protoplanetary discs are misaligned with stellar spins (Batygin 2012). From observations of the RM effect, two misaligned binaries and a dozen of aligned systems have been found (Albrecht et al. 2009, 2014). These observations cover only close binaries with periods less than $100 \mathrm{~d}$. The orbital and tidal evolution of close systems are significantly different from the evolution of wider binaries, making it impossible to extrapolate from these results.

Based on simple simulated observations, we predict that it will require a large, homogeneous sample with precisely known orbital inclinations, orbital velocities and projected rotational velocities to investigate trends in the spin-orbit alignment distribution with the $v \sin i$ method. Such a sample does not currently exist and will be difficult to obtain in the near future. Photometric monitoring surveys (e.g. TESS, Ricker et al. 2015) and future Gaia data releases may help in constraining rotation periods and orbital inclinations. By focusing on eclipsing binaries, the orbital inclination is constrained to $i_{\text {orb }} \sim 90^{\circ}$, eliminating one source of uncertainty. This will however restrict the sample to systems with relatively close orbits. It is similarly possible to eliminate the orbital inclination by measuring the spin-spin alignment in visual binaries. An alternative route is the investi- gation of specific systems in greater detail, for example by identifying the best-studied systems or using alternative techniques such as direct imaging of discs (e.g. Jensen \& Akeson 2014), spectro-interferometry (e.g. Le Bouquin et al. 2009), or measuring alignment via the RM effect (e.g. Albrecht et al. 2007).

Acknowledgements. We thank Alan Hale for useful discussions and acknowledge his significant contributions in pioneering the $v \sin i$ technique by compiling and significantly expanding the sample of binary stars with estimated projected spin-orbit angles. Since 1994, the Hale study has remained the largest and most important study of the spin-orbit alignment of binary stars. We thank the referee for a quick and helpful response. Based on observations made with the Hertzsprung SONG telescope operated on the Spanish Observatorio del Teide on the island of Tenerife by the Aarhus and Copenhagen Universities and by the Instituto de Astrofísica de Canarias. We thank the SONG team members for their helpfulness and quick feedback on the manuscript. We acknowledge support from the Danish Council for Independent Research, through a DFF Sapere Aude Starting Grant no. 4181-00487B. Funding for the Stellar Astrophysics Centre is provided by The Danish National Research Foundation (Grant agreement no.: DNRF106). This research has made use of the Washington Double Star Catalog maintained at the US Naval Observatory. This work has made use of data from the European Space Agency (ESA) mission Gaia (https://www.cosmos.esa.int/gaia), processed by the Gaia Data Processing and Analysis Consortium (DPAC, https://www.cosmos.esa.int/ web/gaia/dpac/consortium). Funding for the DPAC has been provided by national institutions, in particular the institutions participating in the Gaia Multilateral Agreement. This research made use of NumPy (Van Der Walt et al. 2011) and SciPy (Jones et al. 2001). This research made use of Astropy, a communitydeveloped core Python package for Astronomy (Astropy Collaboration 2018). This research made use of matplotlib, a Python library for publication quality graphics (Hunter 2007). This research has made use of the SIMBAD database, operated at CDS, Strasbourg, France (Wenger et al. 2000). This research has made use of NASA's Astrophysics Data System.

\section{References}

Albrecht, S., Reffert, S., Snellen, I., Quirrenbach, A., \& Mitchell, D. S. 2007, A\&A, 474, 565

Albrecht, S., Reffert, S., Snellen, I. A. G., \& Winn, J. N. 2009, Nature, 461, 373 Albrecht, S., Winn, J. N., Carter, J. A., Snellen, I. A. G., \& de Mooij, E. J. W. 2011, ApJ, 726, 68

Albrecht, S., Winn, J. N., Johnson, J. A., et al. 2012, ApJ, 757, 18

Albrecht, S., Setiawan, J., Torres, G., Fabrycky, D. C., \& Winn, J. N. 2013, ApJ, 767,32

Albrecht, S., Winn, J. N., Torres, G., et al. 2014, ApJ, 785, 83

Aly, H., Lodato, G., \& Cazzoletti, P. 2018, MNRAS, 480, 4738

Andersen, M. F., Grundahl, F., Christensen-Dalsgaard, J., et al. 2014, Rev. Mex. Astron. Astrofis. Conf. Ser., 45, 83

Andrade, M. 2019, A\&A, 630, A96

Astropy Collaboration (Price-Whelan, A. M., et al.) 2018, AJ, 156, 123

Bate, M. R. 2015, in Living Together: Planets, Host Stars and Binaries, eds. S. M. Rucinski, G. Torres, \& M. Zejda, ASP Conf. Ser., 496, 37

Bate, M. R. 2018, MNRAS, 475, 5618

Bate, M. R. 2019, MNRAS, 484, 2341

Bate, M. R., Lodato, G., \& Pringle, J. E. 2010, MNRAS, 401, 1505

Batygin, K. 2012, Nature, 491, 418

Blanco-Cuaresma, S., Soubiran, C., Heiter, U., \& Jofré, P. 2014, A\&A, 569, A111

Brinch, C., Jørgensen, J. K., Hogerheijde, M. R., Nelson, R. P., \& Gressel, O. 2016, ApJ, 830, L16

Castelli, F., \& Kurucz, R. L. 2003, in Modelling of Stellar Atmospheres, eds. N.

Piskunov, W. W. Weiss, \& D. F. Gray, IAU Symp., 210, A20

Dawson, R. I., \& Johnson, J. A. 2018, ARA\&A, 56, 175

Donahue, R. A., Saar, S. H., \& Baliunas, S. L. 1996, ApJ, 466, 384

Doyle, A. P., Davies, G. R., Smalley, B., Chaplin, W. J., \& Elsworth, Y. 2014, MNRAS, 444, 3592

Egeland, R. 2018, ApJ, 866, 80

Fabricius, C., Høg, E., Makarov, V. V., et al. 2002, A\&A, 384, 180

Fabrycky, D., \& Tremaine, S. 2007, ApJ, 669, 1298

Fabrycky, D. C., \& Winn, J. N. 2009, ApJ, 696, 1230

Farbiash, N., \& Steinitz, R. 2004, Rev. Mex. Astron. Astrofis. Conf. Ser., 21, 15 Fredslund Andersen, M., Handberg, R., Weiss, E., et al. 2019, PASP, 131, 045003 Gaia Collaboration (Prusti, T., et al.) 2016, A\&A, 595, A1

Gaia Collaboration (Brown, A. G. A., et al.) 2018, A\&A, 616, A1

Gill, S., Maxted, P. F. L., Evans, J. A., et al. 2019, A\&A, 626, A119 
Gray, R. O., \& Corbally, C. J. 1994, AJ, 107, 742

Grevesse, N., Asplund, M., \& Sauval, A. J. 2007, Space Sci. Rev., 130, 105

Griffin, R. F., \& Suchkov, A. A. 2003, ApJS, 147, 103

Grundahl, F., Fredslund Andersen, M., Christensen-Dalsgaard, J., et al. 2017, ApJ, 836, 142

Hale, A. 1994, AJ, 107, 306

Hartkopf, W. I., Mason, B. D., \& Worley, C. E. 2001, AJ, 122, 3472

Hidalgo, S. L., Pietrinferni, A., Cassisi, S., et al. 2018, ApJ, 856, 125

Hodžić, V. K., Triaud, A. H. M. J., Martin, D. V., et al. 2020, MNRAS, 497, 1627

Howe, K. S., \& Clarke, C. J. 2009, MNRAS, 392, 448

Hunter, J. D. 2007, Comput. Sci. Eng., 9, 90

Isaacson, H., \& Fischer, D. 2010, ApJ, 725, 875

Jensen, E. L. N., \& Akeson, R. 2014, Nature, 511, 567

Jensen, E. L. N., Mathieu, R. D., Donar, A. X., \& Dullighan, A. 2004, ApJ, 600, 789

Jones, E., Oliphant, T., Peterson, P., et al. 2001, SciPy: Open Source Scientific Tools for Python

Kennedy, G. M., Matrà, L., Facchini, S., et al. 2019, Nat. Astron., 3, 230

Kervella, P., Arenou, F., Mignard, F., \& Thévenin, F. 2019, A\&A, 623, A72

Konopacky, Q. M., Ghez, A. M., Fabrycky, D. C., et al. 2012, ApJ, 750, 79

Kratter, K. M. 2011, in Evolution of Compact Binaries, eds. L. Schmidtobreick, M. R. Schreiber, \& C. Tappert, ASP Conf. Ser., 447, 47

Kupka, F., Dubernet, M. L., \& VAMDC Collaboration 2011, Balt. Astron., 20, 503

Kurucz, R. L. 1979, ApJS, 40, 1

Kurucz, R. L. 2005, Mem. Soc. Astron. It. Suppl., 8, 14

Le Bouquin, J. B., Absil, O., Benisty, M., et al. 2009, A\&A, 498, L41

Lee, K. I., Dunham, M. M., Myers, P. C., et al. 2016, ApJ, 820, L2

Mamajek, E. E., \& Hillenbrand, L. A. 2008, ApJ, 687, 1264

Mason, B. D., Wycoff, G. L., Hartkopf, W. I., Douglass, G. G., \& Worley, C. E. 2019, VizieR Online Data Catalog: B/wds

Masuda, K., \& Winn, J. N. 2020, AJ, 159, 81

Mayama, S., Pérez, S., Kusakabe, N., et al. 2020, AJ, 159, 12

Monin, J. L., Clarke, C. J., Prato, L., \& McCabe, C. 2007, in Protostars and Planets V, eds. B. Reipurth, D. Jewitt, \& K. Keil, 395
Noyes, R. W., Hartmann, L. W., Baliunas, S. L., Duncan, D. K., \& Vaughan, A. H. 1984, ApJ, 279, 763

Perryman, M. A. C., Brown, A. G. A., Lebreton, Y., et al. 1998, A\&A, 331, 81

Ricker, G. R., Winn, J. N., Vanderspek, R., et al. 2015, J. Astron. Telesc. Instrum. Syst., 1, 014003

Rucinski, S. M. 1992, AJ, 104, 1968

Rucinski, S. 1999, in IAU Colloq. 170: Precise Stellar Radial Velocities, eds. J.

B. Hearnshaw, \& C. D. Scarfe, ASP Conf. Ser., 185, 82

Rucinski, S. M. 2002, AJ, 124, 1746

Schuessler, M., \& Solanki, S. K. 1992, A\&A, 264, L13

Silva Aguirre, V., Davies, G. R., Basu, S., et al. 2015, MNRAS, 452, 2127

Siverd, R. J., Beatty, T. G., Pepper, J., et al. 2012, ApJ, 761, 123

Southworth, J. 2011, MNRAS, 417, 2166

Stassun, K. G., Oelkers, R. J., Paegert, M., et al. 2019, AJ, 158, 138

Sybilski, P., Pawłaszek, R. K., Sybilska, A., et al. 2018, MNRAS, 478, 1942

Thévenin, F., Bigot, L., Kervella, P., et al. 2006, Mem. Soc. Astron. It., 77, 411 Tokovinin, A. 2017, ApJ, 844, 103

Triaud, A. H. M. J., Hebb, L., Anderson, D. R., et al. 2013, A\&A, 549, A18

van Belle, G. T., \& von Braun, K. 2009, ApJ, 694, 1085

Van Der Walt, S., Colbert, S. C., \& Varoquaux, G. 2011, Comput. Sci. Eng., 13, 22

van Saders, J. L., Ceillier, T., Metcalfe, T. S., et al. 2016, Nature, 529, 181 Vaughan, A. H., \& Preston, G. W. 1980, PASP, 92, 385

Wenger, M., Ochsenbein, F., Egret, D., et al. 2000, A\&AS, 143, 9

Williams, J. P., Mann, R. K., Di Francesco, J., et al. 2014, ApJ, 796, 120

Willmarth, D. W., Fekel, F. C., Abt, H. A., \& Pourbaix, D. 2016, AJ, 152, 46

Wilson, O. C. 1968, ApJ, 153, 221

Wilson, O. C. 1978, ApJ, 226, 379

Winn, J. N., Fabrycky, D., Albrecht, S., \& Johnson, J. A. 2010, ApJ, 718, L145

Winn, J. N., Albrecht, S., Johnson, J. A., et al. 2011, ApJ, 741, L1

Worley, C. E., \& Heintz, W. D. 1983, Publ. US Naval Obs. Second Ser., 24, 1

Wright, N. J., Drake, J. J., Mamajek, E. E., \& Henry, G. W. 2011, ApJ, 743, 48

Yee, S. W., Petigura, E. A., \& von Braun, K. 2017, ApJ, 836, 77

Zhou, G., \& Huang, C. X. 2013, ApJ, 776, L35 